\title{
Speed of Pattern Appearance in Reaction-Diffusion Models: Implications in the Pattern Formation of Limb Bud Mesenchyme Cells
}

\author{
TAKASHI MIURA* \\ Department of Human Anatomy and Genetics, \\ University of Oxford, \\ South Parks Road, \\ Oxford OX1 3QX, \\ UK \\ E-mail: Miura@human-anatomy.oxford.ac.uk \\ PHILIP K. MAINI \\ Centre for Mathematical Biology, Mathematical Institute, \\ University of Oxford, \\ 24-29 St Giles, \\ Oxford OX1 3LB, \\ UK \\ E-mail: Maini@maths.ox.ac.uk
}

It has been postulated that fibroblast growth factor (FGF) treatment of cultured limb bud mesenchyme cells reinforces the lateral inhibitory effect, but the cells also show accelerated pattern appearance. In the present study, we analyze how a small change in a specific parameter affects the speed of pattern appearance in a Turing reaction-diffusion system using linear stability analysis. It is shown that the sign of the change in appearance speed is qualitatively decided if the system is under the diffusion-driven instability condition, and this is confirmed by numerical simulations. Numerical simulations also show that a small change in parameter value induced easily detectable differences in the appearance speed of patterns. Analysis of the Gierer-Meinhardt model revealed that a change in a single parameter can explain two effects of FGF on limb mesenchyme cells-reinforcement of lateral inhibition and earlier appearance of pattern. These qualitative properties and easy detectability make this feature a promising tool to elucidate the underlying mechanisms of biological pattern formation where the quantitative parameters are difficult to obtain.

(C) 2003 Society for Mathematical Biology. Published by Elsevier Ltd. All rights reserved.

\section{INTRODUCTION}

Vertebrate limb development is widely studied both experimentally and theoretically [see, for example, the review by Maini and Solursh (1991)]. Most experimen-

\footnotetext{
*Author to whom correspondence should be addressed.

0092-8240/04/040627+23 \$30.00/0 $\quad$ C 2003 Society for Mathematical Biology. Published by Elsevier Ltd. All rights reserved.
} 
tal studies focus on the polarity and subsequent specification of each digit identity. It has been shown that FGF4 and 8 secreted by the apical ectodermal ridge are critical for limb bud outgrowth and proximodistal patterning, while sonic hedgehog secreted from the zone of polarizing activity plays a decisive role in establishing anteroposterior polarity of the limb pattern [for review, see Wolpert (1998), Gilbert (2000)]. On the other hand, theoretical studies concentrate on the periodic aspect of pattern formation using the Turing reaction-diffusion model (Newman and Frisch, 1979; Othmer, 1986; Newman et al., 1988; Downie and Newman, 1995) or its alternatives (Murray, 1993). They are largely ignored by experimental biologists, but recently several reports provide supportive evidence for this periodic property. For example, there exist several polydactylous mutant mice strains which have random digit patterns which are difficult to explain using the one-by-one specification by a positional information mechanism (Hayes et al., 1998). Moreover, the reaggregated limb, which does not have normal anteroposterior gene expression, can form normal digit-like structures (Ros et al., 1994). Based on these findings the idea of periodic pattern formation during limb development is gradually being accepted by experimental biologists [see, for example, Wolpert (1998), Gilbert (2000)].

Since the analysis of periodic pattern in vivo is quite difficult because the domain shape is complex and growing, several researchers utilize the limb micromass culture system for these studies (Owens and Solursh, 1981). In this culture system, limb bud mesenchyme cells are dissociated into separate cells using trypsin, then cultured on a dish at high density. Some cells become chondrocytes while others remain fibroblastic, resulting in a quasi-periodic, stripe-like chondrogenic pattern. Since this final pattern can be directly correlated with the two-dimensional reaction-diffusion pattern, it has been used to study periodic pattern formation in biological systems (Newman, 1996). For example, it has been shown that the wavelength of the pattern can be changed by modulating the diffusion coefficient of the secreted molecules consistent with model predictions (Miura and Shiota, 2000a). It has also been shown that TGF $\beta$ - specifically the TGF $\beta_{2}$ protein-is a good candidate to act as an activator molecule in the reaction-diffusion model in this experimental system (Downie and Newman, 1994; Miura and Shiota, 2000b). (Throughout this paper we define the word 'activator' and 'inhibitor' as those used in a reaction-diffusion system, not the activator or inhibitor of differentiation as used in some experimental papers.)

Recent experimental results address the issue of whether or not speed of pattern appearance in the reaction-diffusion system can be changed by changing a specific interaction within the system. A recent report suggested that fibroblast growth factor (FGF), a signaling molecule that is expressed at the tip of the developing limb bud, can indirectly reinforce the lateral inhibitory effect in limb bud micromass culture (Moftah et al., 2002). Judging from its spatial distribution, FGF itself does not seem to be an inhibitor molecule, but it seems to reinforce the effect of the inhibitor. In the course of re-examining this result, we found that the periodic pattern appears much earlier with FGF treatment [Fig. 1(b)] than in controls 
(a) Control-18h

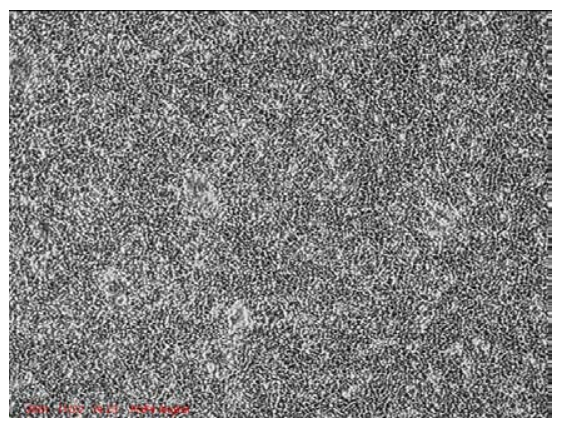

(c) Control-120h

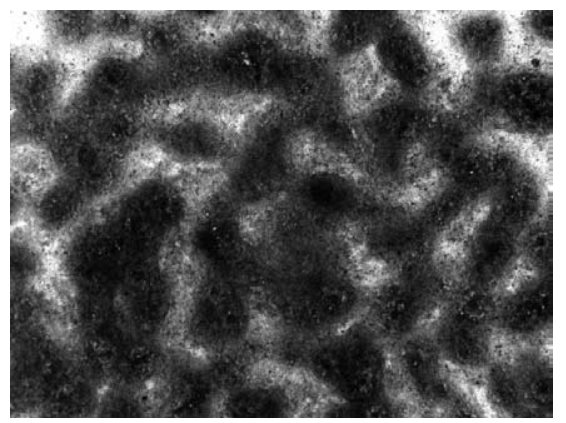

(b) FGF4(+) $18 \mathrm{~h}$

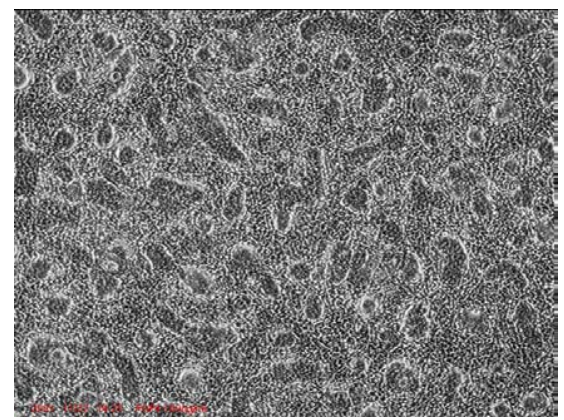

(d) FGF4(+) 120h

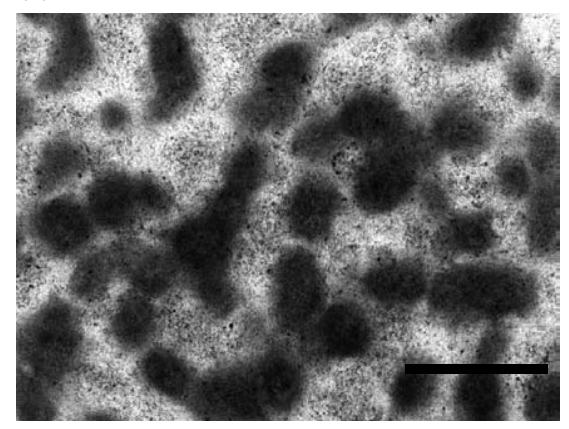

Figure 1. Speed of pattern appearance in cultured limb bud mesenchyme cells of GD10.5 mouse embryos. The method is described in Miura and Shiota (2000a). (a) Control cells cultured for $18 \mathrm{~h}$. Cell distribution is homogeneous and we could not detect any pattern. (b) Cells treated with $100 \mathrm{ng} \mathrm{ml}^{-1}$ FGF4 cultured for $18 \mathrm{~h}$. Cell aggregates form a quasiperiodic pattern, and later become chondrogenic sites. (c) Control cells cultured for 120 h. Chondrogenic cells are stained black by Alcian-blue. (d) FGF4 treated cells cultured for $120 \mathrm{~h}$. The degree of chondrogenic differentiation is suppressed compared with the control. The difference in wavenumber is not as apparent between the control and FGF4 treated cultures. Scale bar, $500 \mu \mathrm{m}$.

[Fig. 1(a)]. This looks puzzling for experimental biologists because even when the appearance of pattern is earlier, final chondrogenic differentiation seems to be suppressed by FGF treatment [Fig. 1(c) and 1(d)]. The descriptive explanation by Moftah et al. (2002) might account for both observations, but we need more rigorous mathematical analysis to see whether these two phenomena can be explained by a mechanism such as the reaction-diffusion model.

We undertook linear stability analysis to see whether a qualitative change in one of the parameters in a Turing reaction-diffusion system results in increase or decrease in the speed of pattern formation. We keep two points in mind to make this analysis biologically relevant. Firstly, we confined our analysis to qualitative characteristics because we cannot obtain all the parameters even in the simple biological system described above. Secondly, we confined our analysis to the linear range because the initial pattern appearance seemed to be quite an early event. 
The cell aggregate pattern first appeared 18-24 h after culture, while the final pattern settled after $96-120 \mathrm{~h}$. In the present study, we analyze the change in the maximum value of the dispersion relation in two types of reaction-diffusion systems, one is the classic activator-inhibitor scheme and the other is the substrate depletion scheme, and confirm the result using numerical calculation. We also undertake detailed analysis of a more realistic nonlinear model and discuss its biological relevance.

\section{Obtaining the Maximum in the Dispersion Relation}

We consider the standard two species reaction-diffusion system as follows:

$$
\begin{aligned}
& \frac{\partial u}{\partial t}=f(u, v)+d_{u} \Delta u \\
& \frac{\partial v}{\partial t}=g(u, v)+d_{v} \Delta v,
\end{aligned}
$$

where $u(x, t)$ and $v(x, t)$ are chemical concentrations at position $x$ and time $t$. Without loss of generality, we set $f(0,0)=g(0,0)=0$ and initial spatial distribution of $u, v$ as $u_{0}$ and $v_{0}$ respectively, which represents the small noise expected in a natural system.

The wavelength of the observed experimental pattern is much smaller than that of the spatial domain, so we assert that the boundary conditions do not exert a strong effect on the final pattern, and take periodic boundary conditions. Here we only consider the linear approximation about the initial value because the appearance of the pattern is quite an early event, as described above. We set

$$
f_{u}=\frac{\partial f}{\partial u}, \quad g_{u}=\frac{\partial g}{\partial u}, \quad f_{v}=\frac{\partial f}{\partial v}, \quad g_{v}=\frac{\partial g}{\partial v}
$$

all evaluated at $(0,0)$ and linearly approximate the equation around $(0,0)$ to give (as usual)

$$
\begin{aligned}
& \frac{\partial u}{\partial t}=f_{u} u+f_{v} v+d_{u} \Delta u \\
& \frac{\partial v}{\partial t}=g_{u} u+g_{v} v+d_{v} \Delta v .
\end{aligned}
$$

We suppose that the parameters in this experimental system are in the diffusiondriven instability range because if we culture cells at very low density and observe each cell separately, which is equivalent to observing the dynamics of the reaction terms only, they remain stable and no chondrogenic differentiation or oscillatory 
(a) Activator-Inhibitor scheme

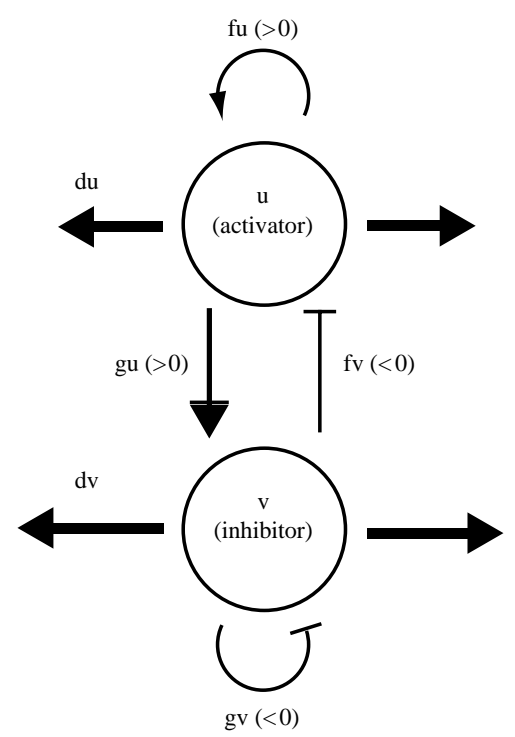

(b) Substrate depletion scheme

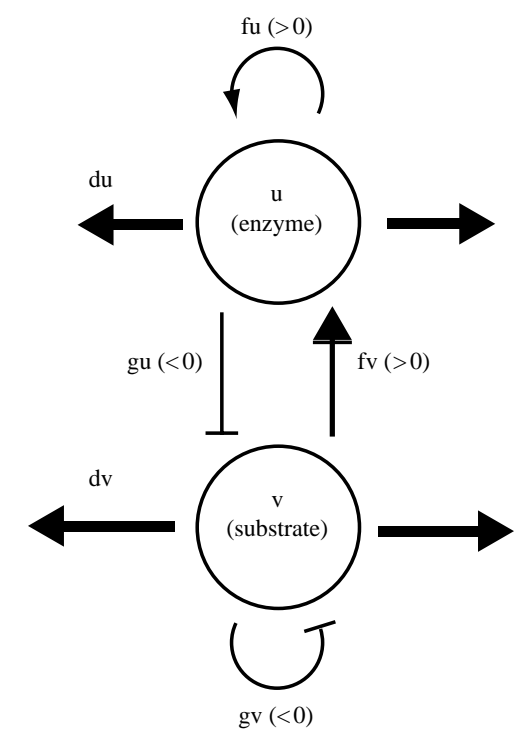

Figure 2. Schematic representations of activator-inhibitor and substrate depletion scheme. Six parameters and their signs are shown.

behavior can be observed. Therefore, these parameters should be within the range described below [see, for example, Murray (1993)]:

$$
\begin{aligned}
& f_{u}+g_{v}<0 \\
& f_{u} g_{v}-f_{v} g_{u}>0 \\
& d_{v} f_{u}+d_{u} g_{v}>0 \\
& \left(d_{v} f_{u}+d_{u} g_{v}\right)^{2}-4 d_{u} d_{v}\left(f_{u} g_{v}-f_{v} g_{u}\right)>0 .
\end{aligned}
$$

In this study we consider both activator-inhibitor and substrate depletion schemes, and the sign of the parameters are as follows: (Fig. 2)

- Activator-inhibitor scheme

- $f_{u}>0$ (activator promotes its own production).

$-f_{v}<0$ (inhibitor inhibits the production of activator).

- $g_{u}>0$ (activator promotes the production of inhibitor).

- $g_{v}<0$ (inhibitor decays).

$-d_{v}>d_{u}$ (inhibitor diffuses faster than activator).

- Substrate depletion scheme (cross-type reaction-diffusion)

- $f_{u}>0$ (enzyme promotes its own production).

$-f_{v}>0$ (enzyme promote its production by substrate). 


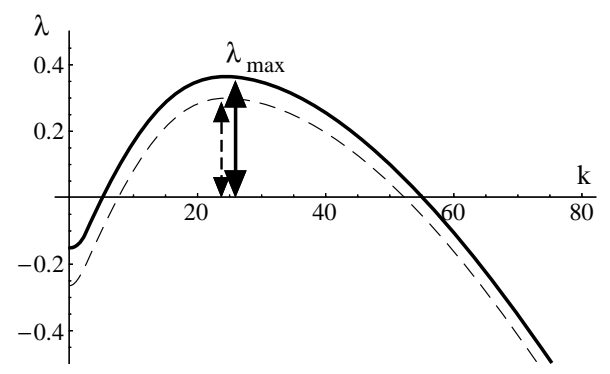

Figure 3. Relationship between pattern appearance speed and dispersion relation. If a slight change in parameter results in an increase in the maximum of the dispersion relation, then the prediction is that the pattern will appear earlier.

- $g_{u}<0$ (substrate is consumed by an enzyme).

- $g_{v}<0$ (enzyme decays).

$-d_{v}>d_{u}$ (substrate diffuses faster than enzyme).

To determine an estimate on the time scale of patterning in the linear regime we set

$$
\mathbf{w}=\left(\begin{array}{c}
u \\
v
\end{array}\right), \quad A=\left(\begin{array}{ll}
f_{u} & f_{v} \\
g_{u} & g_{v}
\end{array}\right), \quad D=\left(\begin{array}{cc}
d_{u} & 0 \\
0 & d_{v}
\end{array}\right)
$$

and the above equation can be simplified to the form below:

$$
\mathbf{w}^{\prime}=A \mathbf{w}+D \Delta \mathbf{w}
$$

where' denotes differentiation with respect to time.

If we consider each wavenumber component separately using the Fourier transform, the solution for each wavenumber component is in general of the form

$$
\mathbf{w} \propto e^{\lambda t} \sin (k x)
$$

Then equation (6) becomes

$$
\left(A-\lambda I-k^{2} D\right) \mathbf{w}=0 .
$$

We require nontrivial solutions for $\mathbf{w}$, so

$$
\begin{aligned}
\operatorname{Det}\left(A-\lambda I-k^{2} D\right) & =\operatorname{Det}\left(\begin{array}{cc}
f_{u}-\lambda-d_{u} k^{2} & f_{v} \\
g_{u} & g_{v}-\lambda-d_{v} k^{2}
\end{array}\right) \\
& =\left(\lambda-f_{u}+d_{u} k^{2}\right)\left(\lambda-g_{v}+d_{v} k^{2}\right)-f_{v} g_{u}=0
\end{aligned}
$$

and hence we have the dispersion relation (see Fig. 3):

$$
\lambda=\frac{1}{2}\left(f_{u}+g_{v}-\left(d_{u}+d_{v}\right) k^{2} \pm \sqrt{4 f_{v} g_{u}+\left(f_{u}-g_{v}+\left(d_{v}-d_{u}\right) k^{2}\right)^{2}}\right) .
$$


Among the two $\lambda$ values, only the higher $\lambda$ value is relevant in this case because under the diffusion-driven instability condition the lower $\lambda$ will always be negative.

This $\lambda$ value becomes positive in a certain range of $k^{2}$, indicating that only certain wavelengths can grow. To obtain the $k^{2}$ for which $\lambda$ has the largest positive value, we differentiate $\lambda$ with respect to $k^{2}$ and equate to zero, that is,

$$
\frac{\partial \lambda}{\partial\left(k^{2}\right)}=\frac{1}{2}\left(-d_{u}-d_{v}+\frac{\left(d_{v}-d_{u}\right)\left(f_{u}-g_{v}+\left(d_{v}-d_{u}\right) k^{2}\right)}{\sqrt{4 f_{v} g_{u}+\left(f_{u}-g_{v}+\left(d_{v}-d_{u}\right) k^{2}\right)^{2}}}\right)=0 .
$$

By solving this equation we obtain

$$
k^{2}=\frac{-d_{u} d_{v}\left(f_{u}-g_{v}\right) \pm\left(d_{u}+d_{v}\right) \sqrt{-d_{u} d_{v} f_{v} g_{u}}}{d_{u} d_{v}\left(d_{v}-d_{u}\right)} .
$$

Looking at the value of $k^{2}$, the denominator should be positive and $-d_{u} d_{v}\left(f_{u}-\right.$ $g_{v}$ ) should be negative in both activator-inhibitor and substrate depletion schemes $\left(f_{u}-g_{v}>0\right)$. Since $k^{2}$ should not be negative, the $k^{2}$ which gives maximum $\lambda$ value is

$$
k^{2}=\frac{-d_{u} d_{v}\left(f_{u}-g_{v}\right)+\left(d_{u}+d_{v}\right) \sqrt{-d_{u} d_{v} f_{v} g_{u}}}{d_{u} d_{v}\left(d_{v}-d_{u}\right)} .
$$

From (13) we can predict that in the physiological parameter range such as $d_{u}, d_{v} \approx 10^{-5} \mathrm{~cm}^{2} \mathrm{~s}^{-1}$ and $f_{u}, f_{v}, g_{u}, g_{v} \approx 10^{-1} \mathrm{~s}^{-1}$ this model will produce a structure with wavelength of order $10^{-1} \mathrm{~cm}$, which is exactly the case in this experimental system.

There are three necessary conditions for this value to be an admissible maximum value. First, since (13) is a square of the wavenumber, this must be larger than 0 , that is,

$$
\frac{\left(d_{u}+d_{v}\right) \sqrt{-d_{u} d_{v} f_{v} g_{u}}-d_{u} d_{v}\left(f_{u}-g_{v}\right)}{d_{u} d_{v}\left(d_{v}-d_{u}\right)}>0 .
$$

As $d_{u}, d_{v}, d_{v}-d_{u}>0$, this condition is simplified as

$$
\left(d_{u}+d_{v}\right) \sqrt{-d_{u} d_{v} f_{v} g_{u}}-d_{u} d_{v}\left(f_{u}-g_{v}\right)>0 .
$$

This condition can be derived from (4), but we use this inequality later for simplicity.

Second, this $k^{2}$ value should give a local $\lambda$ maximum, not minimum. This is easily confirmed.

Third, this wavenumber should be an admissible mode, that is, of the form $k^{2}=\frac{n^{2} \pi^{2}}{L^{2}}$, where $L$ is the domain length and $n$ an integer, to ensure that the boundary conditions are satisfied. To simplify the analysis, we will assume that the $\lambda_{\max }$ we compute below is a reasonable approximation to the growth rate of the admissible mode with maximum growth rate. While not strictly true, this is a good 
approximation for large wavenumber patterns where we change the parameter only slightly.

So we can obtain the maximum $\lambda$ value $\left(\lambda_{\max }\right)$ for the reaction-diffusion system by substituting (10) with (13):

$$
\lambda_{\max }=\frac{d_{v} f_{u}-d_{u} g_{v}-2 \sqrt{-d_{u} d_{v} f_{v} g_{u}}}{d_{v}-d_{u}} .
$$

We can directly obtain the value and make a quantitative prediction if we know all the parameters, but this is almost impossible in a biological experimental system. However, in this case we have an experimental system in which a periodic pattern is formed and we can sometimes detect whether the pattern appears earlier or later by modifying a specific factor. If we can make qualitative predictions from the model as to whether pattern should appear earlier or later by modifying a model parameter in a specific direction, it is possible to qualitatively predict in which pathway in the reaction-diffusion system the factor may be involved.

\section{Effect on $\lambda_{\max }$ of Changing a Specific Parameter}

We assume that, at $(0,0), f_{u}, f_{v}, g_{u}, g_{v}, d_{u}$ and $d_{v}$ are independent parameters and we consider how $\lambda_{\max }$ changes as we vary each parameter in turn, keeping the others fixed.

3.1. Varying $f_{u}$. By simple calculation we obtain

$$
\frac{\partial \lambda_{\max }}{\partial f_{u}}=\frac{d_{v}}{d_{v}-d_{u}} .
$$

$d_{v}>d_{u}>0$, so this value should be positive. Therefore increasing $f_{u}$ always increases the appearance speed.

\subsection{Varying $f_{v}$.}

$$
\frac{\partial \lambda_{\max }}{\partial f_{v}}=\frac{d_{u} d_{v} g_{u}}{\left(d_{v}-d_{u}\right) \sqrt{-d_{u} d_{v} f_{v} g_{u}}} .
$$

The sign of this value is dependent on the sign of $g_{u}$. So in the activator-inhibitor scheme this value should be positive, and in the substrate depletion scheme this value should be negative.

\subsection{Varying $g_{u}$.}

$$
\frac{\partial \lambda_{\max }}{\partial g_{u}}=\frac{d_{u} d_{v} f_{v}}{\left(d_{v}-d_{u}\right) \sqrt{-d_{u} d_{v} f_{v} g_{u}}} .
$$

The sign of this value is dependent on the sign of $f_{v}$. So in the activator-inhibitor scheme this value should be negative, and in the substrate depletion scheme this value should be positive. 


\subsection{Varying $g_{v}$.}

$$
\frac{\partial \lambda_{\max }}{\partial g_{v}}=\frac{-d_{u}}{d_{v}-d_{u}} .
$$

$d_{v}>d_{u}>0$, so this value should be negative. Therefore increasing $g_{v}$ always decreases the appearance speed.

\subsection{Varying $d_{u}$.}

$$
\frac{\partial \lambda_{\max }}{\partial d_{u}}=\frac{d_{u} d_{v}\left(f_{u}-g_{v}\right)-\left(d_{u}+d_{v}\right) \sqrt{-d_{u} d_{v} f_{v} g_{u}}}{d_{u}\left(d_{u}-d_{v}\right)^{2}} .
$$

The denominator is positive, so the sign should be equal to the sign of the numerator. From the inequality (15) presented above, the numerator must obey the following inequality:

$$
-\left(d_{u}+d_{v}\right) \sqrt{-d_{u} d_{v} f_{v} g_{u}}+d_{u} d_{v}\left(f_{u}-g_{v}\right)<0 .
$$

As a result, this value is always negative.

\subsection{Varying $d_{v}$.}

$$
\frac{\partial \lambda_{\max }}{\partial d_{v}}=\frac{\left(d_{u}+d_{v}\right) \sqrt{-d_{u} d_{v} f_{v} g_{u}}-d_{u} d_{v}\left(f_{u}-g_{v}\right)}{d_{v}\left(d_{u}-d_{v}\right)^{2}} .
$$

The denominator is positive, so the sign should be equal to the sign of the numerator. From the inequality (15) presented above, the numerator must obey the following inequality:

$$
\left(d_{u}+d_{v}\right) \sqrt{-d_{u} d_{v} f_{v} g_{u}}-d_{u} d_{v}\left(f_{u}-g_{v}\right)>0 .
$$

As a result, this value is always positive.

These results are summarized in Table 1.

\section{Numerical Calculation}

To confirm the analysis we present numerical simulations for a simple reactiondiffusion model. Currently no quantitative data on the interaction is known, so we use a very simple model here, which only contains linear terms and a cubic term which limits the range of $u$. The model we use is

$$
\begin{aligned}
& \frac{\partial u}{\partial t}=0.6 u-v-u^{3}+0.004 \Delta u \\
& \frac{\partial v}{\partial t}=1.5 u-2 v+0.05 \Delta v
\end{aligned}
$$


Table 1. Summary of change in appearance speed in response to parameter variation in (3). $(+)$ denotes earlier appearance and $(-)$ denotes later appearance as a specific parameter is increased.

\begin{tabular}{ccc}
\hline & \multicolumn{2}{c}{ Change in appearance speed } \\
\cline { 2 - 3 } Parameter varied & Activator-inhibitor scheme & Substrate depletion scheme \\
\hline$f_{u}$ & + & + \\
$f_{v}$ & + & - \\
$g_{u}$ & - & + \\
$g_{v}$ & - & - \\
$d_{u}$ & - & - \\
$d_{v}$ & + & + \\
\hline
\end{tabular}

for the activator-inhibitor scheme and

$$
\begin{aligned}
& \frac{\partial u}{\partial t}=0.5 u+0.6 v-u^{3}+0.004 \Delta u \\
& \frac{\partial v}{\partial t}=-1.9 u-2 v+0.05 \Delta v
\end{aligned}
$$

for the substrate depletion scheme.

We undertook one-dimensional simulations on a domain of size $2 \pi$ with periodic boundary conditions. Initial conditions in each case are random white noise about the equilibrium point, i.e., $f(u, v)=g(u, v)=0$ and the magnitude of initial white noise is of order $1.0 \times 10^{-3}$.

From Section 3 it is predicted that an increase in $f_{u}, f_{v}$ or $d_{v}$ results in the earlier appearance of the pattern and an increase in $g_{u}, g_{v}$ or $d_{u}$ results in later appearance of the pattern in the activator-inhibitor scheme. To confirm this, we visualized the time evolution of the pattern of the control system and 'experimentally treated' system where one of these six parameters is increased by $10 \%$. All of them seem to reach steady state after $t=200$, but the speed of appearance of the pattern is quite different among these groups. It is observed that an increase in $f_{u}, f_{v}$ and $d_{v}$ results in earlier appearance of the pattern and an increase in $g_{u}, g_{v}$ and $d_{u}$ results in later appearance of the pattern (Fig. 4), which is consistent with the mathematical prediction. On the other hand, in the substrate depletion scheme, an increase in $f_{u}$, $g_{u}$ and $d_{v}$ results in the earlier appearance of the pattern and an increase in $f_{v}, g_{v}$ and $d_{v}$ results in later appearance of the pattern (Fig. 5), which is also consistent with the mathematical prediction.

The difference in the speed of pattern appearance seems quite obvious in all cases and usually we can detect the difference by a single run of numerical calculation. To confirm the result above is not a specific case, we carried out the simulation 40 times each with different initial conditions and automatically detected the time required for the difference between highest and lowest concentration of $u$ to be 0.1 . Statistically significant differences are detected among all the treated and control 

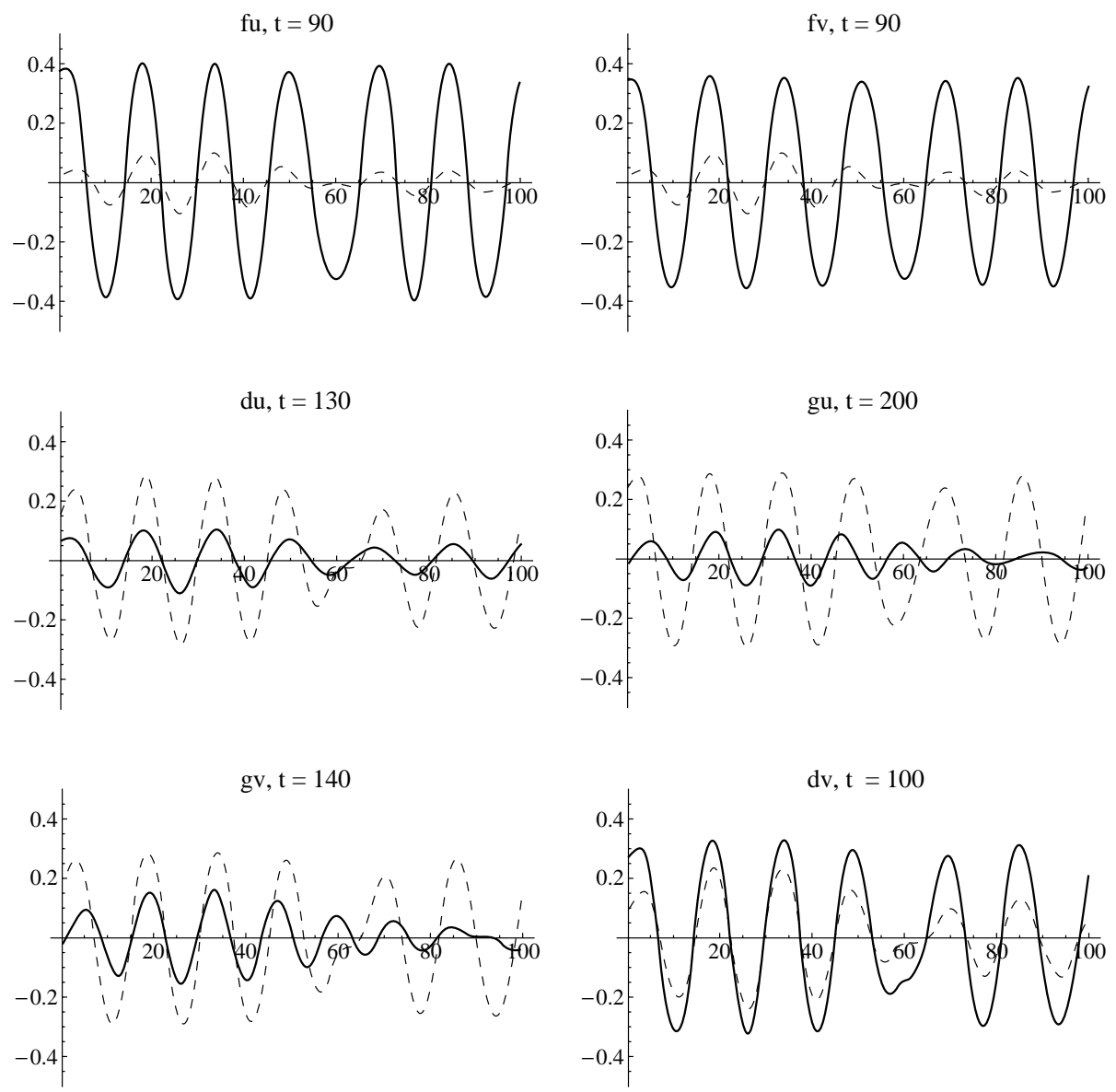

Figure 4. Speed of pattern appearance in activator-inhibitor scheme (25). The simulation is undertaken on the domain $(0,2 \pi)$ with periodic boundary conditions. We set the initial condition as white random noise whose magnitude is of order $1.0 \times 10^{-3}$ and simulate until $t=200$. The modified parameter and observed time is described at the top of each graph. Dashed lines represent the original pattern of $u$ distribution and the thick, continuous lines represent the pattern in which one specific parameter is increased by $10 \%$. From these pictures we can see that increasing $f_{u}, f_{v}$ or $d_{v}$ results in earlier appearance of the pattern and increasing $g_{u}, g_{v}$ or $d_{u}$ results in later appearance of the pattern.

groups, and their directions are the same as predicted by the mathematical analysis (Fig. 6).

Moreover, it is possible to analytically predict how much the time required for the pattern to reach a certain amplitude will change by varying a specific parameter. Suppose we change parameters such that $\lambda_{\max }$ in (16) changes from $\lambda_{\max 1}$ to $\lambda_{\max 2}$ and fix the initial and detection threshold amplitude of $u$ as $u_{0}$ and $u_{\text {threshold }}$ respectively. Then if the time required for the pattern to reach a certain amplitude is $t_{1}$ and $t_{2}$, respectively,

$$
u_{\text {threshold }}=u_{0} e^{\lambda_{\max 1} t_{1}}=u_{0} e^{\lambda_{\max 2} t_{2}} .
$$



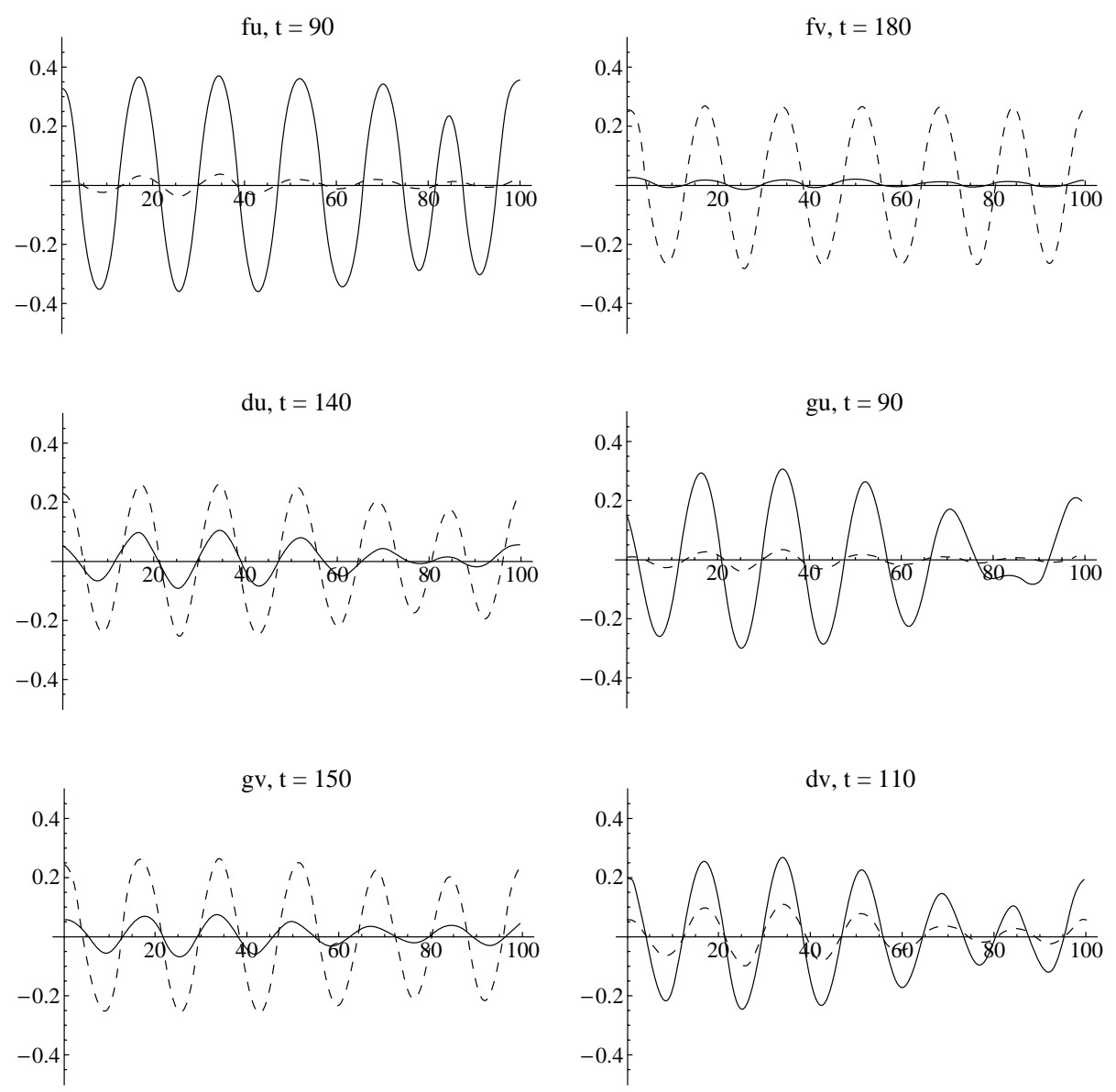

Figure 5. Speed of pattern appearance in substrate depletion scheme (26). The simulation is undertaken on the domain $(0,2 \pi)$ with periodic boundary conditions. We set the initial condition as white random noise whose magnitude is of order $1.0 \times 10^{-3}$ and simulate until $t=200$. The modified parameter and observed time is described at the top of each graph. Dashed lines represent the original pattern of $u$ distribution and the thick, continuous lines represent the pattern in which one specific parameter is increased by $10 \%$. From these pictures we can see that increasing $f_{u}, g_{u}$ and $d_{v}$ results in earlier appearance of the pattern and increasing $f_{v}, g_{v}$ or $d_{u}$ results in later appearance of the pattern.

\section{Hence we obtain}

$$
t_{2}=\frac{\lambda_{\max 1}}{\lambda_{\max 2}} t_{1}
$$

We calculate the predicted time by analytically obtaining $\lambda_{\max 1} / \lambda_{\max 2}$ and numerically obtaining the control value $\left(t_{1}\right)$. As can be seen in Fig. 6, the numerical calculation results agree very closely with the analytical predictions. In conclusion, the numerical calculation results are consistent with the mathematical analysis described above. 
(a) Activator-inhibitor scheme (25)

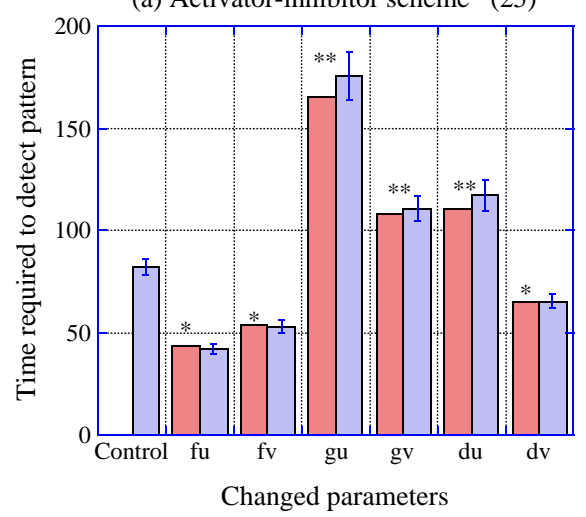

(b) Substrate-depletion scheme

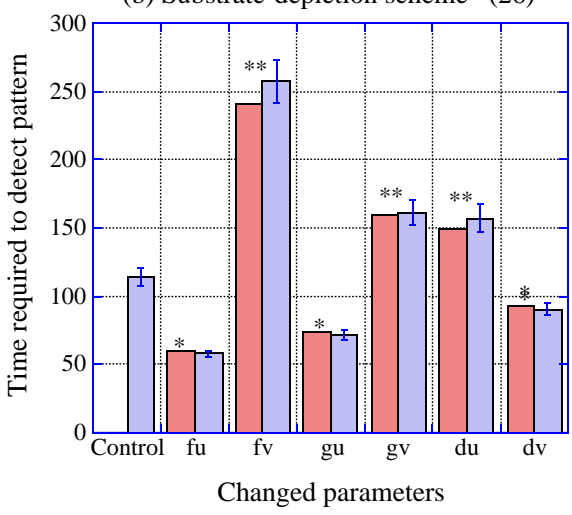

$\square$ Analytical prediction

* $\quad$ Pattern appears earlier, $\mathrm{p}<0.01$

Simulation result

Figure 6. Statistical analysis of the speed of pattern appearance in the activator-inhibitor (25) and substrate depletion (26) scheme. The simulation is for the domain $(0,2 \pi)$ with periodic boundary conditions. Numerical simulation with a specific parameter set was carried out 40 times each with different random initial distribution. We set the magnitude of the random noise to be of order $1.0 \times 10^{-3}$. The time required for the amplitude of pattern to reach the pattern detection threshold (which is set to $1.0 \times 10^{-1}$, a considerably smaller value than the final amplitude of the pattern) is automatically detected. Statistically significant differences between control and treated groups are detected by analysis of variance (ANOVA) and the direction of difference is identical to the mathematical predictions from Section 3. The time required to detect the pattern by varying a specific parameter is analytically predicted and shown next to the numerically obtained value.

\section{Analysis OF THE GIERER-Meinhardt TyPE REACTION DIFFUSION MODEL}

5.1. The model. The analysis above can be useful to determine what will happen when a specific interaction is blocked or promoted, but in actual experimental conditions the interactions are sometimes cross-linked and it is unrealistic to consider that the 4 linear reaction term parameters are independent. Here we undertake detailed analysis of the Gierer-Meinhardt type reaction-diffusion model (Gierer and Meinhardt, 1972), which is widely utilized in modeling various biological pattern formation phenomena.

The equations we use are

$$
\begin{aligned}
& \frac{\partial u}{\partial t}=k_{1}-k_{2} u+k_{3} \frac{u^{2}}{v}+d_{u} \Delta u \\
& \frac{\partial v}{\partial t}=k_{4} u^{2}-k_{5} v+d_{v} \Delta v,
\end{aligned}
$$


Table 2. Summary of change in appearance speed in response to parameter variation in (29). (+) denotes earlier appearance and (-) denotes later appearance as a specific parameter is increased. $( \pm)$ indicates that the appearance speed depends on other parameters.

\begin{tabular}{cc}
\hline Parameter varied & Change in appearance speed \\
\hline$k_{1}$ & - \\
$k_{2}$ & + \\
$k_{3}$ & + \\
$k_{4}$ & - \\
$k_{5}$ & \pm \\
\hline
\end{tabular}

where $k_{1}, k_{2}, k_{3}, k_{4}, k_{5}>0$. The analysis is similar to that above (details given in the appendix) and a summary of the results is presented in Table 2.

\subsection{Numerical calculation-both reinforcement of lateral inhibition and ear-} lier pattern appearance can be induced by a single parameter change. We carried out exactly the same numerical calculation as in Section 4 using the GiererMeinhardt type reaction-diffusion system. We undertook one-dimensional simulations on a domain of size $2 \pi$ with periodic boundary conditions. Initial conditions in each case are random white noise about the equilibrium point (A.1) and the magnitude of initial white noise is of order $1.0 \times 10^{-3}$. From appendix it is predicted that increasing $k_{2}$ or $k_{3}$ results in the earlier appearance of the pattern while increasing $k_{1}$ or $k_{4}$ results in later appearance of the pattern. The effect of $k_{5}$ should depend on the parameter set. To confirm this, we calculated the time evolution of the pattern of the control system and 'experimentally treated' system where one of these five parameters is increased by $10 \%$, and automatically detected the time required for the pattern amplitude to reach the pattern detection threshold, which is set to $1.0 \times 10^{-1}$. The results, shown in Fig. 7, are consistent with the analytical predictions.

From the above analytical results, that involving $k_{1}$ is of special interest for us because it represents the base production of the activator and increasing $k_{1}$ should result in higher final activator concentration. However, the analytical result showed that increasing $k_{1}$ results in a slowing down of the pattern appearance speed. Therefore, decreasing $k_{1}$ will both explain promoted lateral inhibition and earlier pattern appearance. To see whether this is the case we undertook numerical calculation to assay both initial pattern appearance speed and final concentration of $u$. We first set $\left(k_{1}, k_{2}, k_{3}, k_{4}, k_{5}, d_{u}, d_{v}\right)=(0.1,0.3,0.2,0.4,0.7,0.02,0.8)$, and changed $k_{1}$ from 0.3 to 0.0 and compared the results. Fig. 8 shows that in the initial phase of morphogenesis the case with $k_{1}=0.0$ forms the pattern much earlier than the $k_{1}=0.3$ case, but finally the activator concentration in the latter attains a higher value on average. To show this is not a special case caused by specific initial conditions, we again carried out 40 simulations with different white noise initial conditions. The time required to detect the pattern is significantly shorter and average $u$ (activator) concentration is statistically lower under low $k_{1}$. 

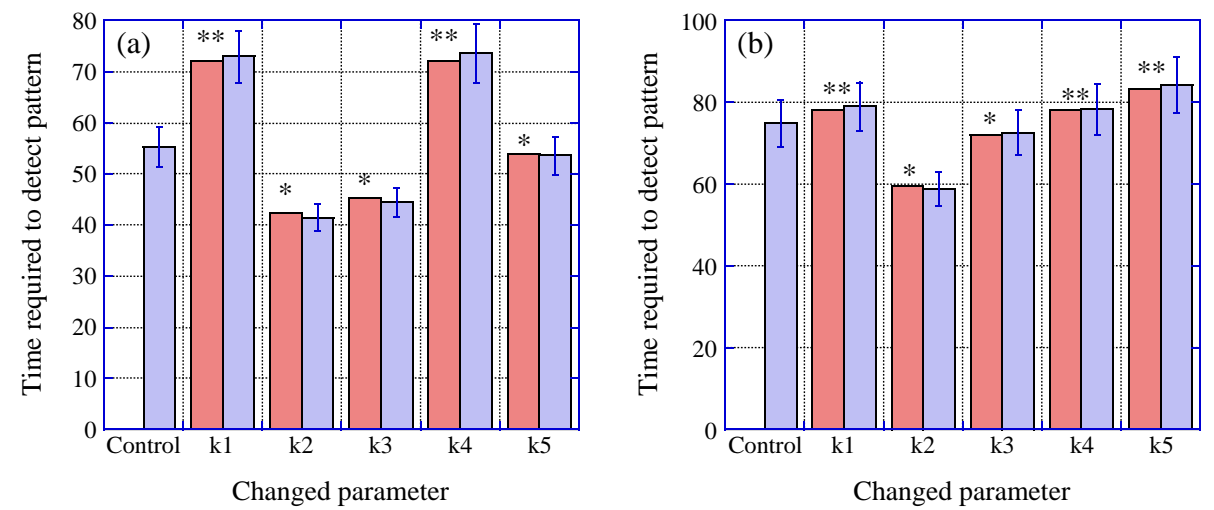

$$
\begin{aligned}
& \square \text { Analytical prediction } \\
& \text { Simulation result }
\end{aligned}
$$

Figure 7. Statistical analysis of the speed of pattern appearance in the Gierer-Meinhardt reaction-diffusion system (29). Numerical calculation with a specific parameter set was carried out 300 times each with different random initial distribution. We set the magnitude of the random noise to be of order $1.0 \times 10^{-3}$. The simulations are carried out on the domain $(0,2 \pi)$ with periodic boundary conditions. The time required for the amplitude of pattern to reach the pattern detection threshold (which we set to $1.0 \times 10^{-1}$, considerably smaller than the final amplitude of the pattern) is automatically detected. (a) $\left(k_{1}, k_{2}, k_{3}, k_{4}, k_{5}, d_{u}, d_{v}\right)=(0.1,0.3,0.2,0.4,0.7,0.02,0.8)$, where $\partial \lambda_{\max } / \partial k_{5}>0$. (b) $\left(k_{1}, k_{2}, k_{3}, k_{4}, k_{5}, d_{u}, d_{v}\right)=(0.3,0.4,0.8,0.1,0.6,0.02,0.4)$, where $\partial \lambda_{\max } \partial k_{5}<0$. A statistically significant difference is detected between control and treated groups by ANOVA and the direction of difference is identical to the mathematical predictions from appendix. The time required to detect the pattern by varying a specific parameter is analytically predicted and shown next to the numerically obtained value.

\section{Discussion}

In the present study, we mathematically analyzed how the speed of pattern appearance in the very early phase of pattern formation is affected by modification of specific linear terms of the reaction-diffusion system, and confirmed the analytical prediction using numerical simulation. In summary, in the activator-inhibitor scheme, increasing $f_{u}$ or $f_{v}$ causes earlier appearance of the pattern and increasing $g_{u}$ or $g_{v}$ results in delay of pattern emergence. In the substrate depletion scheme (cross-type reaction-diffusion system), increasing $f_{u}$ or $g_{u}$ results in earlier appearance of the pattern, while increasing $f_{v}$ or $g_{v}$ results in later appearance of the pattern. In both reaction types, increasing $d_{u}$ results in later appearance and increasing $d_{v}$ results in earlier appearance of the pattern.

We have to be careful when using $\lambda_{\max }$ as a measure of pattern appearance speed because in some cases the boundary condition can affect the result. For example, in the case when the wavelength of the pattern is similar to the length of the spatial domain, boundary conditions may restrict admissible wavenumbers to values which are away from the peak of the dispersion relation. In that case we have 
(a)

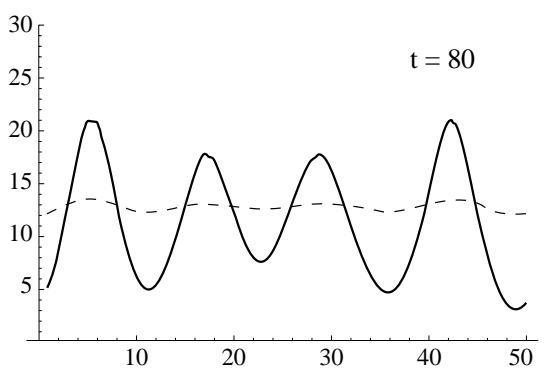

(b)

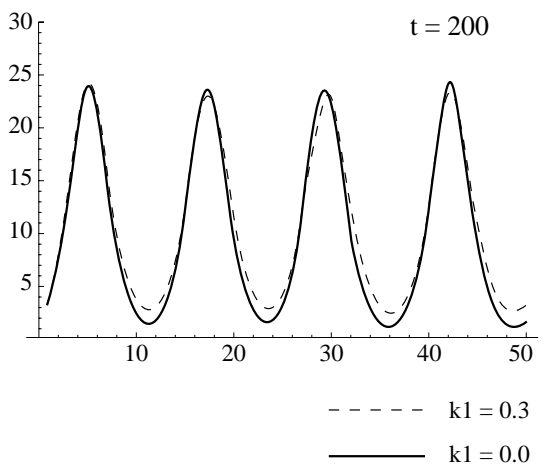

(c) Time required to detect pattern

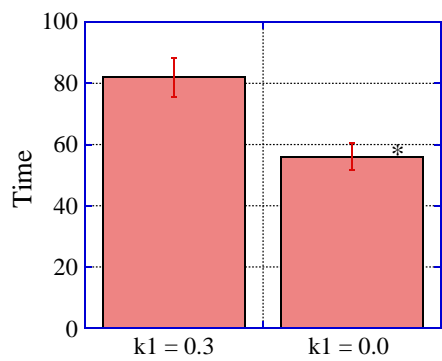

(d) Average activator value of final pattern

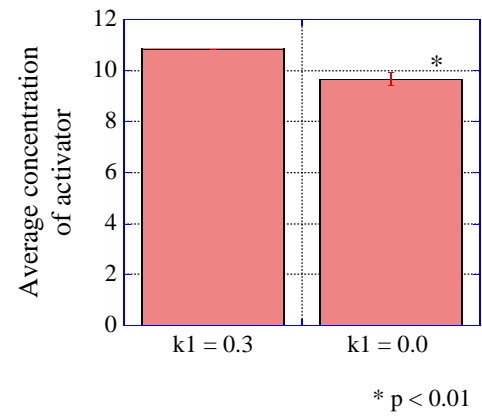

Figure 8. Results of numerical simulations of the Gierer-Meinhardt model (29), which may represent FGF treatment of the limb bud mesenchyme cells. The numerical simulation details are identical to Fig. 7(b) except for the value of $k_{1}$. (a) Result of numerical calculation at the onset of pattern formation. The spatial distribution of $u$ at $t=80$ is shown. (b) Final pattern (at $t=200$ ) obtained by numerical calculation. The spatial distribution of $u$ concentration is shown. (c) Statistical analysis of the speed of pattern appearance in the Gierer-Meinhardt system. A total of 40 numerical simulations for each parameter set were carried out with different random initial distribution. We set the magnitude of the random noise to be of order $1.0 \times 10^{-3}$. The time required for the amplitude of the pattern to reach the pattern detection threshold (which we set to $1.0 \times 10^{-1}$, considerably smaller than the final amplitude of pattern) is automatically detected. A statistically significant difference is detected between control and treated groups (Student $t$-test) and the direction of difference is identical to the mathematical prediction in appendix. (d) Statistical analysis of the average concentration of $u$. A statistically significant difference is detected between control and treated groups (Student $t$-test).

to differentiate (10) for $k$ constant to see whether the pattern at the admissible wavenumbers will appear earlier or later. Interestingly, it is possible to decide the sign of $\partial \lambda / \partial f_{u}, \partial \lambda / \partial f_{v}, \partial \lambda / \partial g_{u}, \partial \lambda / \partial g_{v}, \partial \lambda / \partial d_{u}, \partial \lambda / \partial d_{v}$ in all $k$, which means that by modifying one specific linear parameter the dispersion relation curve simply goes up or down and no intersection of two dispersion relation curves occurs. This is not true when we modify two or more parameters simultaneously, where intersection can happen. 
The result of the mathematical analysis in the activator-inhibitor scheme can be descriptively explained as follows: for the rate of initial pattern appearance, a positive feedback loop of activator is crucially important. So a change that reinforces the positive feedback loop results in the earlier appearance of the pattern, and a change that suppresses the positive feedback loop results in the later appearance of the pattern. For diffusion coefficients, faster activator diffusion coefficient blocks the activator positive feedback because diffusion is inhibitory for peak formation. A larger inhibitor diffusion coefficient results in the suppression of inhibitor peaks at the same places as activator peaks, which reinforces the activator positive feedback loop. Therefore increasing $d_{u}$ results in later pattern appearance and increasing $d_{v}$ results in earlier appearance of the pattern. Concerning our original question, in the activator-inhibitor scheme there is no parameter range in which a single linear parameter change results in both a reinforcing of the lateral inhibitory effect and the earlier appearance of the pattern.

However, analysis of the Gierer-Meinhardt model shows that a single parameter change does result in both a reinforcing of the lateral inhibitory effect and earlier appearance of the pattern. In this model, decreasing $k_{1}$, which means decreasing the basal production of activator and hence a relative reinforcement of the lateral inhibitory effect, will always result in the speeding up of pattern appearance. This seems to be counterintuitive at first, but the qualitative explanation of this phenomenon is as follows: when we increase $k_{1}$, we increase the equilibrium concentration. The positive feedback reaction of the activator nears saturation and results in decreased $f_{u}$ around the equilibrium point. In this case, the average of $u$ is increased while the amplitude of spatial $u$ distribution is decreased, which results in higher $u$ concentration at the 'valley' of the pattern but not at the peak. If we assume the concentration of $u$ corresponds to chondrogenic differentiation, this is exactly what is happening in the actual experimental system (Fig. 1). It is important to note that the term 'more differentiated' from an experimental point of view can mean, from a theoretical interpretation, either an increase in amplitude of the morphogen concentration and/or an upward shift in its baseline value.

Obviously we have to keep in mind that there are other interpretations for the experimental and analytical results. The experimental result may simply reflect the existence of two independent mechanisms - periodic pattern formation and pattern formation speed — and FGF might affect both of them. Previous hypotheses suggest that pattern formation occurs only in the progress zone (Newman and Frisch, 1979), and FGF might play a role in enabling the reaction-diffusion system there. And the above analysis does not suggest that $k_{1}$ in the Gierer-Meinhardt system is the only candidate for the FGF effect. If we use different models some parameters may play the same role. For example, if we add a constant term in the first equation of (3) it may work the same as $k_{1}$.

Analysis of how changes in $d_{u}$ and $d_{v}$ affect model predictions can be useful in determining how the diffusion term arises in actual experimental systems. The molecular nature of the activator and inhibitor remains to be elucidated and it is not 
certain whether the 'diffusion' term in the reaction-diffusion model actually reflects diffusion of some secretory molecules. For example, this could be achieved by the random motion of cells, which is actually observed in experimental systems we are currently considering (Miura et al., 2000). Since the diffusion coefficient of activator and inhibitor should be considerably different to form a stable pattern, it is possible that these diffusion terms are implemented by completely different mechanisms in actual biological systems. So if random cell movement is involved for one of the diffusion terms, suppressing cell movement by altering the rate of cell movement by modifying adhesion between cell and extracellular matrix (Delannet et al., 1994) can result in either earlier or later appearance of the pattern. Thus we could distinguish whether random cell movement is involved in the diffusion term of activator or inhibitor by observing the time course of the formation of the pattern.

The limb bud micromass culture system is a promising system to elucidate the underlying mechanisms of periodic pattern formation in biological systems. The cells form a two-dimensional chondrogenic pattern, and they show various morphological properties which can be experimentally manipulated. For example, we can modify the wavenumber of the pattern (Miura and Shiota, 2000a; Miura et al., 2000), or speed of appearance of the pattern as described above. The culture system also exhibits stripe-spot transitions using retinoic acid (data not shown), which can be mathematically explained using nonlinear analysis (Ermentrout, 1991; Lyons and Harrison, 1992). A combination of experimental methods and mathematical analysis as presented in this paper will, we hope, contribute to understanding the mechanisms of pattern formation in actual biological systems.

\section{ACKNOWLEDGEMENTS}

The authors are grateful for the technical assistance of Professor Gillian MorrissKay and her lab members in the Department of Human Anatomy and Genetics in the University of Oxford, and Drs Edmund Crampin and Anotida Madzvamuse in the Centre for Mathematical Biology, Mathematical Institute, Oxford, for useful discussions. Part of this work was supported by the Japan Society for the Promotion of Science.

\section{APPEndix A: Detailed ANAlysis OF THE GiERER-MeinhardT TYPE REACTION-DIFFUSION MODEL}

In the Gierer-Meinhardt system (29) the equilibrium point is

$$
(u, v)=\left(\frac{k_{3} k_{5}+k_{1} k_{4}}{k_{2} k_{4}}, \frac{\left(k_{3} k_{5}+k_{1} k_{4}\right)^{2}}{k_{2}^{2} k_{4} k_{5}}\right),
$$


and the linearization around this point yields

$$
\begin{aligned}
& f_{u}=\frac{k_{2}\left(k_{3} k_{5}-k_{1} k_{4}\right)}{k_{3} k_{5}+k_{1} k_{4}} \\
& f_{v}=-\frac{k_{2}^{2} k_{3} k_{5}^{2}}{\left(k_{3} k_{5}+k_{1} k_{4}\right)^{2}} \\
& g_{u}=\frac{2\left(k_{3} k_{5}+k_{1} k_{4}\right)}{k_{2}} \\
& g_{v}=-k_{5} .
\end{aligned}
$$

If we substitute (A.2) into (16), we obtain

$$
\lambda_{\max }=\frac{d_{u} k_{5}\left(k_{3} k_{5}+k_{1} k_{4}\right)-d_{v} k_{2}\left(k_{3} k_{5}-k_{1} k_{4}\right)-2 k_{5} \sqrt{2 d_{u} d_{v} k_{2} k_{3}\left(k_{3} k_{5}+k_{1} k_{4}\right)}}{\left(d_{v}-d_{u}\right)\left(k_{3} k_{5}+k_{1} k_{4}\right)} .
$$

A.1. Diffusion-driven instability condition in the Gierer-Meinhardt model. From (4) we can obtain the following inequalities for diffusion-driven instability:

$$
\begin{aligned}
& k_{3} k_{5}\left(k_{2}-k_{5}\right)-k_{1} k_{4}\left(k_{2}+k_{5}\right)<0 \\
& d_{v} k_{2}\left(k_{3} k_{5}-k_{1} k_{4}\right)>d_{u} k_{5}\left(k_{3} k_{5}+k_{1} k_{4}\right)>0 \\
& d_{v}^{2} k_{2}^{2}\left(k_{3} k_{5}-k_{1} k_{4}\right)^{2}+d_{u}^{2} k_{5}^{2}\left(k_{3} k_{5}+k_{1} k_{4}\right)^{2} \\
& \quad-2 d_{u} d_{v} k_{2} k_{5}\left(k_{3} k_{5}+k_{1} k_{4}\right)\left(3 k_{3} k_{5}+k_{1} k_{4}\right)>0 .
\end{aligned}
$$

A.2. Varying $\boldsymbol{k}_{\mathbf{1}}$. By simple calculation we obtain

$$
\frac{\partial \lambda_{\max }}{\partial k_{1}}=\frac{-2 d_{v} k_{2} k_{3} k_{4} k_{5}+k_{4} k_{5} \sqrt{2 d_{u} d_{v} k_{2} k_{3}\left(k_{3} k_{5}+k_{1} k_{4}\right)}}{\left(d_{v}-d_{u}\right)\left(k_{3} k_{5}+k_{1} k_{4}\right)^{2}} .
$$

$d_{v}>d_{u}>0$, so the denominator is positive. The first term of the numerator is negative and the second term is positive, so the sign of the numerator should be identical to

$$
\begin{aligned}
\left(-2 d_{v} k_{2} k_{3} k_{4} k_{5}+k_{4} k_{5} \sqrt{2 d_{u} d_{v} k_{2} k_{3}\left(k_{3} k_{5}+k_{1} k_{4}\right)}\right) \\
\quad \times\left(2 d_{v} k_{2} k_{3} k_{4} k_{5}+k_{4} k_{5} \sqrt{2 d_{u} d_{v} k_{2} k_{3}\left(k_{3} k_{5}+k_{1} k_{4}\right)}\right) \\
=2 d_{v} k_{2} k_{3} k_{4}^{2} k_{5}\left(d_{u} k_{5}\left(k_{3} k_{5}+k_{1} k_{4}\right)-2 d_{v} k_{2} k_{3} k_{5}\right) \\
<2 d_{v} k_{2} k_{3} k_{4}^{2} k_{5}\left(d_{v} k_{2}\left(k_{3} k_{5}-k_{1} k_{4}\right)-2 d_{v} k_{2} k_{3} k_{5}\right) \\
=2 d_{v} k_{2} k_{3} k_{4}^{2} k_{5}\left(-d_{v} k_{2} k_{3} k_{5}-d_{v} k_{1} k_{2} k_{4}\right)<0 .
\end{aligned}
$$

Therefore increasing $k_{1}$ always decreases the appearance speed. 
A.3. Varying $\boldsymbol{k}_{2}$. By simple calculation we obtain

$$
\frac{\partial \lambda_{\max }}{\partial k_{2}}=\frac{d_{v} k_{2}\left(k_{3} k_{5}-k_{1} k_{4}\right)-k_{5} \sqrt{2 d_{u} d_{v} k_{2} k_{3}\left(k_{3} k_{5}+k_{1} k_{4}\right)}}{\left(d_{v}-d_{u}\right) k_{2}\left(k_{3} k_{5}+k_{1} k_{4}\right)} .
$$

$d_{v}>d_{u}>0$, so the denominator is positive. So the sign of the value is identical to

$$
d_{v} k_{2}\left(k_{3} k_{5}-k_{1} k_{4}\right)-k_{5} \sqrt{2 d_{u} d_{v} k_{2} k_{3}\left(k_{3} k_{5}+k_{1} k_{4}\right)} .
$$

From (A.5), $d_{v} k_{2}\left(k_{3} k_{5}-k_{1} k_{4}\right)>0$. So the first term of this expression is positive and the second term is negative. Therefore, the sign of this expression is identical to

$$
\begin{aligned}
\left(d_{v} k_{2}(\right. & \left.\left.k_{3} k_{5}-k_{1} k_{4}\right)-k_{5} \sqrt{2 d_{u} d_{v} k_{2} k_{3}\left(k_{3} k_{5}+k_{1} k_{4}\right)}\right) \\
& \times\left(d_{v} k_{2}\left(k_{3} k_{5}-k_{1} k_{4}\right)+k_{5} \sqrt{2 d_{u} d_{v} k_{2} k_{3}\left(k_{3} k_{5}+k_{1} k_{4}\right)}\right) \\
= & d_{v}^{2} k_{2}^{2}\left(k_{3} k_{5}-k_{1} k_{4}\right)^{2}-2 d_{u} d_{v} k_{2} k_{3} k 5^{2}\left(k_{3} k_{5}+k_{1} k_{4}\right) \\
> & -\left(d u^{2} k^{5}\left(k_{3} k_{5}+k_{1} k_{4}\right)^{2}-2 d_{u} d_{v} k_{2} k_{5}\left(k_{3} k_{5}+k_{1} k_{4}\right)\left(3 k_{3} k_{5}+k_{1} k_{4}\right)\right) \\
& -2 d_{u} d_{v} k_{2} k_{3} k_{5}^{2}\left(k_{3} k_{5}+k_{1} k_{4}\right) \\
= & -d_{u} k_{5}\left(k_{3} k_{5}+k_{1} k_{4}\right)\left(d_{u} k_{5}\left(k_{3} k_{5}+k_{1} k_{4}\right)-2 d_{v} k_{2}\left(2 k_{3} k_{5}+k_{1} k_{4}\right)\right) \\
> & -d_{u} k_{5}\left(k_{3} k_{5}+k_{1} k_{4}\right)\left(d_{v} k_{2}\left(k_{3} k_{5}-k_{1} k_{4}\right)-2 d_{v} k_{2}\left(2 k_{3} k_{5}+k_{1} k_{4}\right)\right) \\
= & 3 d_{u} d_{v} k_{2} k_{5}\left(k_{3} k_{5}+k_{1} k_{4}\right)\left(k_{3} k_{5}+k_{1} k_{4}\right) \\
> & 0 .
\end{aligned}
$$

Therefore $\partial \lambda_{\max } / \partial k_{2}$ is always positive and increasing $k_{2}$ always increases the pattern appearance speed.

A.4. Varying $\boldsymbol{k}_{3}$. By simple calculation we obtain

$$
\frac{\partial \lambda_{\max }}{\partial k_{3}}=\frac{k_{1} k_{4}\left(2 d_{v} k_{2} k_{3} k_{5}-k_{5} \sqrt{2 d_{u} d_{v} k_{2} k_{3}\left(k_{3} k_{5}+k_{1} k_{4}\right)}\right)}{\left(d_{v}-d_{u}\right) k_{3}\left(k_{3} k_{5}+k_{1} k_{4}\right)^{2}} .
$$

From $d_{v}>d_{u}$, the denominator is positive. So the sign of this value is identical to

$$
2 d_{v} k_{2} k_{3} k_{5}-k_{5} \sqrt{2 d_{u} d_{v} k_{2} k_{3}\left(k_{3} k_{5}+k_{1} k_{4}\right)}
$$


The first term of this expression is positive and the second term is negative. So the sign of this expression is identical to

$$
\begin{aligned}
\left(2 d_{v}\right. & \left.k_{2} k_{3} k_{5}-k_{5} \sqrt{2 d_{u} d_{v} k_{2} k_{3}\left(k_{3} k_{5}+k_{1} k_{4}\right)}\right) \\
& \times\left(2 d_{v} k_{2} k_{3} k_{5}+k_{5} \sqrt{2 d_{u} d_{v} k_{2} k_{3}\left(k_{3} k_{5}+k_{1} k_{4}\right)}\right) \\
= & 2 d_{v} k_{2} k_{3} k_{5}\left(2 d_{v} k_{2} k_{3} k_{5}-d_{u} k_{5}\left(k_{3} k_{5}+k_{1} k_{4}\right)\right) \\
> & 2 d_{v} k_{2} k_{3} k_{5}\left(2 d_{v} k_{2} k_{3} k_{5}-d_{v} k_{2}\left(k_{3} k_{5}-k_{1} k_{4}\right)\right) \\
= & 2 d_{v}^{2} k_{2}^{2} k_{3} k_{5}\left(k_{3} k_{5}+k_{1} k_{4}\right) \\
> & 0 .
\end{aligned}
$$

Therefore $\partial \lambda_{\max } / \partial k_{3}$ is always positive and increasing $k_{3}$ always increases the pattern appearance speed.

\section{A.5. Varying $\boldsymbol{k}_{\mathbf{4}}$.}

$$
\frac{\partial \lambda_{\max }}{\partial k_{4}}=\frac{-2 d_{v} k_{1} k_{2} k_{3} k_{5}+k_{1} k_{5} \sqrt{2 d_{u} d_{v} k_{2} k_{3}\left(k_{3} k_{5}+k_{1} k_{4}\right)}}{\left(d_{v}-d_{u}\right)\left(k_{3} k_{5}+k_{1} k_{4}\right)^{2}} .
$$

The denominator is positive $\left(d_{v}>d_{u}\right)$, and the first term of the numerator is negative and the second term is positive. So the sign of this expression is identical to

$$
\begin{aligned}
\left(-2 d_{v}\right. & \left.k_{1} k_{2} k_{3} k_{5}+k_{1} k_{5} \sqrt{2 d_{u} d_{v} k_{2} k_{3}\left(k_{3} k_{5}+k_{1} k_{4}\right)}\right) \\
& \times\left(2 d_{v} k_{1} k_{2} k_{3} k_{5}+k_{1} k_{5} \sqrt{2 d_{u} d_{v} k_{2} k_{3}\left(k_{3} k_{5}+k_{1} k_{4}\right)}\right) \\
= & 2 d_{v} k_{1}^{2} k_{2} k_{3} k_{5}\left(-2 d_{v} k_{2} k_{3} k_{5}+d_{u} k_{5}\left(k_{3} k_{5}+k_{1} k_{4}\right)\right) \\
< & 2 d_{v} k_{1}^{2} k_{2} k_{3} k_{5}\left(-2 d_{v} k_{2} k_{3} k_{5}+d_{v} k_{2}\left(k_{3} k_{5}-k_{1} k_{4}\right)\right) \\
= & -2 d_{v}^{2} k_{1}^{2} k_{2}^{2} k_{3} k_{5}\left(k_{3} k_{5}+k_{1} k_{4}\right)<0 .
\end{aligned}
$$

Therefore $\partial \lambda_{\max } / \partial k_{4}$ is always negative and increasing $k_{4}$ always decreases the pattern appearance speed.

\section{A.6. Varying $\boldsymbol{k}_{5}$.}

$$
\frac{\partial \lambda_{\max }}{\partial k_{5}}=\frac{\left(2 d_{v} k_{1} k_{2} k_{3} k_{4}+d_{u}\left(k_{3} k_{5}+k_{1} k_{4}\right)^{2}\right)-\left(2 k_{1} k_{4}+k_{3} k_{5}\right) \sqrt{2 d_{u} d_{v} k_{2} k_{3}\left(k_{3} k_{5}+k_{1} k_{4}\right)}}{\left(d_{v}-d_{u}\right)\left(k_{3} k_{5}+k_{1} k_{4}\right)^{2}} .
$$


We cannot determine easily the sign of this expression. For example, when $\left(k_{1}\right.$, $\left.k_{2}, k_{3}, k_{4}, k_{5}, d_{u}, d_{v}\right)=(0.5,1.0,0.9,0.4,0.9,0.01,0.2), \quad \partial \lambda_{\max } / \partial k_{5}=0.0476677$ $>0$. However, when $\left(k_{1}, k_{2}, k_{3}, k_{4}, k_{5}, d_{u}, d_{v}\right)=(0.3,0.4,0.8,0.1,0.6,0.02,0.4)$, $\partial \lambda_{\max } / \partial k_{5}=-0.148849<0$. Both of the parameter sets satisfy the diffusiondriven instability conditions.

\section{REFERENCES}

Delannet, M., F. Martin, B. Bossy, D. A. Cheresh, L. F. Reichardt and J. L. Duband (1994). Specific roles of the alpha $\mathrm{v}$ beta 1 , alpha $\mathrm{v}$ beta 3 and alpha $\mathrm{v}$ beta 5 integrins in avian neural crest cell adhesion and migration on vitronectin. Development 120, 2687-2702.

Downie, S. A. and S. A. Newman (1994). Morphogenetic differences between fore and hind limb precartilage mesenchyme: relation to mechanisms of skeletal pattern formation. Dev. Biol. 162, 195-208.

Downie, S. A. and S. A. Newman (1995). Different roles for fibronectin in the generation of fore and hind limb precartilage condensations. Dev. Biol. 172, 519-530.

Ermentrout, B. (1991). Stripes or spots? Nonlinear effects in bifurcation of reactiondiffusion equations on the square. Proc. R. Soc. Lond. A 434, 413-417.

Gierer, A. and H. Meinhardt (1972). A theory of biological pattern formation. Kybernetik 12, 30-39.

Gilbert, S. F. (2000). Developmental Biology, Massachusettes: Sinauer.

Hayes, C., M. F. Lyon and G. M. Morriss-Kay (1998). Morphogenesis of doublefoot (dbf), a mouse mutant with polydactyly and craniofacial defects. J. Anat. 193, 81-91.

Lyons, M. J. and L. G. Harrison (1992). Stripe selection: an intrinsic property of some pattern-forming models with nonlinear dynamics. Dev. Dyn. 195, 201-215.

Maini, P. K. and M. Solursh (1991). Cellular mechanisms of pattern formation in the developing limb. Int. Rev. Cytol. 129, 91-133.

Miura, T., M. Komori and K. Shiota (2000). A novel method for analysis of the periodicity of chondrogenic patterns in limb bud cell culture: correlation of in vitro pattern formation with theoretical models. Anat. Embryol. (Berl) 201, 419-428.

Miura, T. and K. Shiota (2000a). Extracellular matrix environment influences chondrogenic pattern formation in limb bud micromass culture: experimental verification of theoretical models. Anat. Rec. 258, 100-107.

Miura, T. and K. Shiota (2000b). Tgfbeta2 acts as an "activator" molecule in reactiondiffusion model and is involved in cell sorting phenomenon in mouse limb micromass culture. Dev. Dyn. 217, 241-249.

Moftah, M. Z., S. A. Downie, N. B. Bronstein, N. Mezentseva, J. Pu, P. A. Maher and S. A. Newman (2002). Ectodermal fgfs induce perinodular inhibition of limb chondrogenesis in vitro and in vivo via fgf receptor 2. Dev. Biol. 249, 270-282.

Murray, J. D. (1993). Mathematical Biology, Berlin: Springer.

Newman, S. A. (1996). Sticky fingers: Hox genes and cell adhesion in vertebrate limb development. Bioessays 18, 171-174.

Newman, S. A. and H. L. Frisch (1979). Dynamics of skeletal pattern formation in developing chick limb. Science 205, 662-668.

Newman, S. J., H. L. Frisch and J. K. Percus (1988). On the stationary state analysis of reaction-diffusion mechanisms for biological pattern formation. J. Theor. Biol. 134, 183-197. 
Othmer, H. G. (1986). On the Newman-Frisch model of limb chondrogenesis. J. Theor. Biol. 121, 505-508.

Owens, E. M. and M. Solursh (1981). In vitro histogenic capacities of limb mesenchyme from various stage mouse embryos. Dev. Biol. 88, 297-311.

Ros, M. A., G. E. Lyons, S. Mackem and J. F. Fallon (1994). Recombinant limbs as a model to study homeobox gene regulation during limb development. Dev. Biol. 166, 59-72.

Wolpert, L. (1998). Principles of Development, Oxford University Press.

Received 3 July 2003 and accepted 25 September 2003 